\title{
Geometric Inductor Breaks Resistance Quantum “Limit”
}

\author{
A geometric superinductor made of a tightly wound aluminum wire can \\ achieve an impedance about 5 times larger than a hypothesized \\ fundamental limit.
}

By Erika K. Carlson

A superinductor is an inductor with an electrical impedance that exceeds $6.45 \mathrm{k} \Omega$, a value set by Planck's constant and the charge of an electron. It was thought that inductors could only exceed this value-known as the resistance quantum - through a mechanism called kinetic inductance, which is the inductance from a material's intrinsic properties. Now, Matilda Peruzzo, Andrea Trioni, and colleagues of the Institute of Science and Technology Austria show another route to high impedance by building a geometric superinductor-where the superinductance comes instead from the material's geometric arrangement [1].

Kinetic inductance can come from several types of material properties. For example, granular aluminum can act as a kinetic inductor because grain boundaries in the material slow down-or impede-current. Geometric inductance, on the other hand, boosts impedance through the shape or pattern that a material is molded into. A coiled wire, for example, has a greater

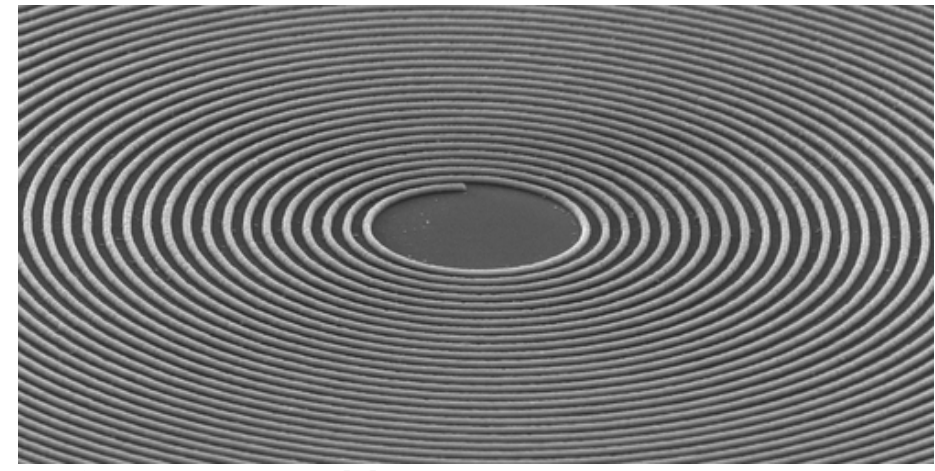

Credit: M. Peruzzo et al. [1] impedance than a straight wire because the magnetic field of each loop acts on other loops to raise the overall impedance.

To achieve geometric superinductance, Peruzzo, Trioni, and colleagues nanofabricated very tightly wound spirals of thin aluminum wire on a silicon-based substrate. They then removed as much of the substrate as they could, creating a nearly free-standing coil. They made more than a hundred such devices with various geometries. Ultimately, they achieved impedances of up to 5 times the resistance quantum for ac currents with frequencies as high as a few gigahertz, showing unequivocally that neither the resistance quantum nor the impedance of free space-another hypothesized limit for geometric inductors-is actually a fundamental limit.

Erika K. Carlson is a Corresponding Editor for Physics based in New York City.

\section{REFERENCES}

1. M. Peruzzo et al., "Surpassing the resistance quantum with a geometric superinductor," Phys. Rev. Applied 14, 044055 (2020). 